\title{
Dissociable Self Effects for Emotion Regulation: A Study of Chinese Major Depressive Outpatients
}

\author{
Xiaoxia Wang, ${ }^{1}$ Zhengzhi Feng, ${ }^{1}$ Daiquan $\mathrm{Zhou}^{2}{ }^{2} \mathrm{Xu} \mathrm{Lei},{ }^{3}$ \\ Tongquan Liao, ${ }^{4} \mathrm{Li}$ Zhang, ${ }^{1} \mathrm{Bing} \mathrm{Ji}^{2}{ }^{2}$ and Jing $\mathrm{Li}^{2}$ \\ ${ }^{1}$ Department of General Psychology, College of Psychology, Third Military Medical University, Street 30, \\ Gaotanyan of Shapingba District, Chongqing 400038, China \\ ${ }^{2}$ Radiology Department, Southwest Hospital, Third Military Medical University, Chongqing 400037, China \\ ${ }^{3}$ School of Psychology, Southwest University, Chongqing 400715, China \\ ${ }^{4}$ Xin Qiao Hospital, Third Military Medical University, Chongqing 400038, China
}

Correspondence should be addressed to Zhengzhi Feng; fengzhengzhi@gmail.com

Received 9 September 2013; Revised 19 February 2014; Accepted 6 March 2014; Published 3 April 2014

Academic Editor: Yong He

Copyright (C) 2014 Xiaoxia Wang et al. This is an open access article distributed under the Creative Commons Attribution License, which permits unrestricted use, distribution, and reproduction in any medium, provided the original work is properly cited.

\begin{abstract}
Reappraisal is an adaptive emotion regulation strategy while the role of self-perspective in reappraisal process of depressed patients is largely unknown in terms of goals (valence/arousal) and tactics (detachment/immersion). In this study, 12 depressed individuals and 15 controls were scanned with MRI during which they either attend naturally to emotional stimuli, or adopt detachment/immersion strategy. Behaviorally, no group differences in self-reported emotion regulation effectiveness were found. In addition, we observed that (1) patients were less able to downregulate amygdala activation with recruitment of more dorsal lateral prefrontal cortex (dlPFC) when adopting detachment strategy regardless of valence, and this preserved ability to regulate emotion was inversely associated with severity of symptoms; (2) patients had deficits in upregulating amygdala activation when adopting immersion strategy, with less inferior frontal gyrus (IFG) activation and strengthening coupling of dlPFC and ventral medial prefrontal cortex (vmPFC) with amygdala; (3) comparison between groups yielded that patients showed stronger vmPFC activation under either self-detached or self-immersed condition. In conclusion, impaired modulatory effects of amygdala in depressed patients are compensated with strengthening cognitive control resources, with dissociable effects for different self-perspectives in reappraisal. These results may help clarify the role of self-perspective underlying reappraisal in major depression.
\end{abstract}

\section{Introduction}

According to the fourth edition of Diagnostic and Statistical Manual of Mental Disorders (DSM-IV), anhedonia and/or a lingering low mood are the defining characteristics of major depressive disorder (MDD). A body of evidence implies a trait-like role of maladaptive emotion regulation strategies in MDD vulnerability [1], among which the strategy of reappraisal has received the most extensive attention. In clinical settings, reappraisal plays a pivotal role in cognitive behavioral therapy (CBT) interventions [2] that predicts resilience in depressed patients [3]. Converging neuroimaging evidence indicates that reappraisal engages the lateral and medial sectors of the dorsal/ventral prefrontal cortex and subcortical structures such as amygdala. Notably, these structures are also foci of brain network dysfunction in the neurological models of depression $[4,5]$ which propose that attenuated top-down cognitive control networks are companied with unrestrained activation in emotional regions (i.e., amygdala) [6-9]. Reappraisal may involve the utilization of cognitive control to regulate semantic representations of affective stimuli which in turn attenuate amygdala reactivity [10]. Moreover, reappraisal is generally viewed as an adaptive emotion regulation strategy which is dysfunctional in depression with less frequency of daily use [11] as well as abnormal neural activation patterns $[9,12,13]$. Therefore, reappraisal may be a promising target for disclosing the vulnerable characteristics of depression.

Operationally, reappraisal refers to a combination of approaches that require generation, maintenance, coordination of top-down cognitive reinterpretation, and bottom-up 
appraisal of affective events and monitoring them in working memory over time [14]. Reappraisal strategies can vary in their goals (what is regulated) and tactics (how is emotion regulated), leading to multiple variants of experiment paradigm [15].

The circumplex model of affect suggests that all emotions can be distinguished in terms of varying levels of valence and arousal, with two distinct neural systems mediating the representation of affective states. It is addressed that common as well as distinct neural substrates underlie the regulation of different valences of emotion [16]. Primarily, there could be two reappraisal goals (what people want to achieve) that effectively regulate negative emotion: to upregulate positive emotion and downregulate negative emotion [15]. Positive emotion can be used to counter negative emotion in psychologically resilient individuals [17], spiral upward toward positive reappraisals, and transform negative affect into positive affect, leading to optimal functioning [18]. However, most studies of reappraisal in major depression focus on decreasing negative emotion, possibly due to excessive negative affect prevalent in MDD. To date only two studies to our knowledge explore positive emotion regulation in MDD, and they adopt different reappraisal working definitions. One study reports that depressed individuals fail to sustain activation in neural circuits underlying positive affect [19]. The other study finds no significant differences in downregulating positive affect by reappraisal between normal controls and MDD [12]. Neither study simultaneously investigates both processes, making it open to question whether only one or both processes are impaired. Positive and negative affect may facilitate the use of different sources of information, in terms of relation between self and situation [20]. Nonetheless, restriction to one valence makes it difficult to generalize the conclusion about reappraisal in common emotional state.

Another critical question remains to be answered is what is regulated. Reappraisal can be self- or situation-oriented [21]. The former focuses on reinterpreting the personal meaning of the emotional object to make it more or less self-relevant, while the latter focuses on reinterpreting the consequence or the reality of emotional stimuli without changing one's relationship to the stimuli [22]. In some studies, self-oriented reappraisal with decreasing affect as the regulating goal is also called detached reappraisal [23-25] or distancing reappraisal [15], which is efficient for emotion regulation [26]. In these studies, reappraisal is conceptualized as taking an objective or third-person perspective upon the emotional stimuli/situation. Reappraisal had been the target of clinical research on stress coping and CBT long before the conduction of laboratory experimental research on reappraisal as a form of emotion regulation. In line with this tradition, other researchers with social and clinical background deem self-distancing as a form of self-reflection and introduce psychological distance to distinguish adaptive versus maladaptive self-reflection [26-29]. Recent evidence has linked effectiveness of self-distancing to adaptive behavioral outcomes [27]. It is reported that depressed individuals can benefit from analyzing negative emotion events from a self-distancing perspective [28]. Moreover, evaluative rather than experiential self-referential processing is inherent in depression [30]. However, the modulatory effect of selffocused processing on reappraisal has been deemphasized. One feasible approach is to validate the role of self-focused processing in reappraisal and to manipulate reappraisal strategies along this dimension.

The purpose of this study was to examine the neural mechanisms of self-related reappraisal in Chinese MDD outpatients. Block designs were employed to avoid naturally declining emotion processes when watching emotionally arousing pictures. Emotion control areas such as dorsal lateral prefrontal cortex (dlPFC) and ventral medial prefrontal cortex (vmPFC) were selected as regions of interest since these two areas seemed to be involved in pathogenesis of depression and influence the expression of depressive symptoms [4, 31]. Amygdala was examined because it could act as a neural proxy for changes in emotion induction [22]. We hypothesized that (1) self-related neural networks underlying reappraisal would be differentially activated in depressed patients versus controls; (2) major depression would show abnormal neural activations underlying selfrelated reappraisal of affect.

\section{Methods}

2.1. Participants. Twelve unmedicated major depressed outpatients and 15 normal controls were recruited. The patients were diagnosed through a structured clinical interview according to DSM-IV. For depressed patients, an inclusion criterion with current depressed episode was adopted, according to the DSM-IV. All patients were assessed with SDS, BDI, and HAMD before participating into experiment, with mild to moderate depression symptoms (HAMD $\geq 18$; $\mathrm{BDI} \geq 14$; SDS $\geq 35$ ). For healthy controls, semi-standard interviews were conducted and assessed with SDS and BDI, with no current depressed mood (BDI $<4$ or SDS $<50)$. For both groups, exclusion criteria were history of neurological disease or presence of axis I psychiatric disorders, psychiatric medication use within the last two weeks, or implanted cardiac or ferrous metal devices. The patients and normal controls were also assessed with BDI/SDS and the patients with HAMD (24-item version) additionally. Statistically significant differences were found in BDI and SDS scores (Table 1). A written informed consent was obtained from all subjects before experiment. This study was approved by the Ethics Committee of the Third Military Medical University.

2.2. Experimental Procedure. The participants were trained on a computer during a previous session to get familiar with the reappraisal strategies they were to use during the scan. They were instructed to either attend to the visual stimuli or reappraise (self-detached versus self-immersed) their emotion reactions to each picture. Tasks were performed in three consecutive sessions after acquisition of structural images. Emotional stimuli were selected from the International Affective Pictures System (IAPS) [32] and matched for content of scenes and people as well as valence and arousal for each condition (mean valence $(V)$ and arousal (A): neutral/attend, $V=5.06, A=2.74$; positive/attend, 
TABLE 1: Demographic and clinical data of MDD and normal control groups.

\begin{tabular}{lccc}
\hline Measure & MDD & Control & Sig. \\
& Mean/SD $(n=12)$ & Mean/SD $(n=15)$ & $7 / 8$ \\
Gender ratio (male/female) & $5 / 7$ & $25.80(5.89)$ & 0.55 \\
Age & $29.50(8.46)$ & $14.80(2.83)$ & 0.07 \\
Years of education & $14.00(3.77)$ & right $(15)$ & 0.53 \\
Handedness & right (12) & $4.27(4.23)$ & - \\
BDI & $26.17(12.65)$ & $36.54(5.74)$ & 0.00 \\
SDS & $64.08(12.60)$ & & 0.00 \\
HAMD & $25.23(4.97)$ & - \\
\hline
\end{tabular}

Note: BDI: beck depression inventory; SDS: self-rating depression scale. Both groups were matched for age, sex ratio, and years of education.

$V=7.17, A=5.45$; negative/attend, $V=2.34, A=5.60$; positive/detached, $V=7.26, A=5.69$; negative/detached, $V=2.69, A=5.82$; positive/immersed, $V=6.94, A=$ 5.30; negative/immersed, $V=2.42, A=5.09$ ). One-way ANOVA for stimuli in each session resulted in insignificant differences in arousal when taking valence as a factor (all $P s>0.05)$. Twelve pictures were used for each valence under each condition and one more neutral picture for the start of each condition. The neutral picture was eliminated from MRI data analysis afterwards to prevent from signal drift.

For the attend condition (session 1), subjects should simply view the picture without taking efforts to alter their emotion; for the self-immersed conditions (session 2), subjects should perceive each picture as real and engage themselves in the situation depicted, by imagining themselves or a loved one in the scene; for the self-detached conditions (session 3), subjects should view the situation as fake or unreal and detach themselves from the situation. The attend condition was set as the control condition. Participants were told not to close their eyes or direct eyes away from the pictures during each trial and be able to relax during the break after each trial (Figure 1).

During the scanning, stimuli were projected onto a screen, reflected by a mirror in front of the subjects. The task was performed in three consecutive sessions ("maintain," "detach," and "immerse"), in the order of the last two sequentially counterbalanced across all subjects. The instruction for each condition was given at the beginning of each block. Each trial consisted of four components: fixation, induction or regulation, rating, and rest. A fixation cross was displayed for $2 \mathrm{~s}$, and then an IAPS picture appeared for $8 \mathrm{~s}$ during which subjects either simply viewed or reappraised the picture, followed by an affect rating screen $(1=$ no intensity to $4=$ very intense), and a black blank screen was shown for $8 \mathrm{~s}$ for relaxation. A four-point scale was chosen because it forces the subjects to make emotional judgments and was proved to be reliable for emotion discrimination in a previous study [33]. Affect ratings were collected using a two-button response box held in each hand. After experiment, all the subjects were inquired to confirm the effectiveness of emotion regulation.

2.3. MRI Data Acquisition. MRI data were collected on a Siemens 3T Allegra MRI scanner. A high-resolution T1weighted 3D image (T1WI) was acquired, with slice thickness

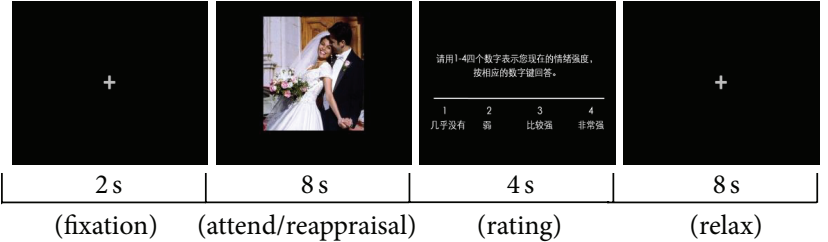

FIGURE 1: Sequence of a trials in attend/reappraisal task. Instructions were given prior to each block.

$=4 \mathrm{~mm}$, field of view $(\mathrm{FOV})=240 \times 240 \times 240 \mathrm{~mm}^{3}$, and matrix $=256 \times 256 \times 256$. Functional images were obtained from 30 gradient-echo T2* -weighted slices (slice thickness $=4 \mathrm{~mm}$ ) per volume. A single shot gradient-recalled echoecho planar imaging (SS-GRE-EPI) sequence was used with a time repetition of $2000 \mathrm{~ms}$, a flip angle of $90^{\circ}$, time echo of $30 \mathrm{~ms}$, FOV of $240 \times 240 \mathrm{~mm}^{2}$, matrix of $64 \times 64$, slice thickness of $4 \mathrm{~mm}$, and slice interval of $0.8 \mathrm{~mm}$. For coregistration, 176 sagittal whole-brain scans were collected by 3-D magnetization-prepared rapid gradient-echo imaging (MPRAGE), with $\mathrm{TR}=1970 \mathrm{~ms}$, TE $=3.93 \mathrm{~ms}$, a flip angle = $15^{\circ}$, slice thickness $=1.70 \mathrm{~mm}$, slice interval $=0.85 \mathrm{~mm}, \mathrm{FOV}$ $=250 \times 250 \mathrm{~mm}^{2}$, and a matrix $=448 \times 512$.

\subsection{Data Analysis}

2.4.1. Self-Report Data. The emotional state ratings during the experiment were analyzed with PASW (Version 19, SPSS Inc., Chicago, IL, USA). A two-way ANOVA was conducted to analyze the effect of the emotional picture presentation (negative, neutral, positive) on emotional state in the viewing condition. A $2 \times 3 \times 2$ repeated-measures ANOVA including the factors group (MDD, HC), valence (negative, positive), and condition (attend, self-detachment, self-immersion) was calculated to illuminate the effects of regulation on emotional state. The neutral condition was neglected for the second analysis as there was no neutral picture in the reappraisal condition.

2.4.2. Functional MRI Data. Data were preprocessed and statistically analyzed with SPM 8 (http://www.fil.ion.ucl.ac.uk/ spm/software/spm8/) and Matlab 7.8.0 (Math Works, Natick, 
MA). The preprocessing included realignment, spatial normalization, and spatial smoothing $(8 \mathrm{~mm})$.

GLM Analysis. The first level analysis consisted of seven regressors (attend neutral, attend positive, attend negative, decrease positive, decrease negative, increase positive, and increase negative) modeled with a duration of 8 seconds convolved with the hemodynamic response function. A highpass filter was applied and six head motion parameters were included as residuals. In a second level analysis, we conducted a repeated measures general linear model (GLM) with emotion and reappraisal as within-subjects factors and group as a between-subjects factor. Post hoc $t$ tests were then performed to examine contrasts between factors with significant main effects and interactions. Significant difference of statistical maps for whole brain analysis was set at $P<0.05$, corrected for multiple comparisons using cluster-size thresholding (54 voxels $^{2}$ ) based on Monte Carlo simulation.

We then performed region of interest (ROI) analyses upon a priori region of interest implicated in emotion reactivity and regulation (bilateral dlPFC, vmPFC, and amygdala). If vmPFC and dlPFC are critical neural substrates for pathogenesis of depression, then damage to either area should affect the expression of depressive symptoms. We used anatomical masks based on the Talairach daemon database, defined by WFU Pick atlas software (version 3.0; ANSIR Laboratory, WFU School of Medicine, Winston-Salem, NorthCarolina), and set the threshold at $P<0.05$ with an extent threshold of 5 voxels [34]. As to thresholding, the incorporation of extent threshold into $P$ value effectively achieved equivalent correction for multiple comparisons [35]. ROI time courses were extracted within anatomically defined ROIs by generating the first eigenvariate of $8 \mathrm{~mm}$ around the peak voxels using a Matlab package REX (Response Exploration) [36]. Eigenvariates were extracted and global-scaled to produce a time series of functional data in units of percent signal change referenced to the SPM default intracerebral mean of 100.

Psychophysiological Interaction Analysis. This analysis was performed to identify the brain regions that produce a downregulating effect on the amygdala during emotion regulation. A $10 \mathrm{~mm}$ seed region around the peak activation in bilateral amygdala was identified when we contrasted reappraisal and attends condition for each valence between two groups. Time series were extracted for each subject as the first regressor (physiological variable). The second regressor represented psychological variable (condition parameter). The regressor of interest was the interaction between the physiological variable and psychological variable, created from product time series of VOI and the condition parameter. We then created subtraction contrast between conditions of interests, and all individual contrast images were included into a second-level group random-effects analysis, in which taskdependent effects were investigated using a two-sample $t$ test for two groups. Significant activations exhibiting PPI-related
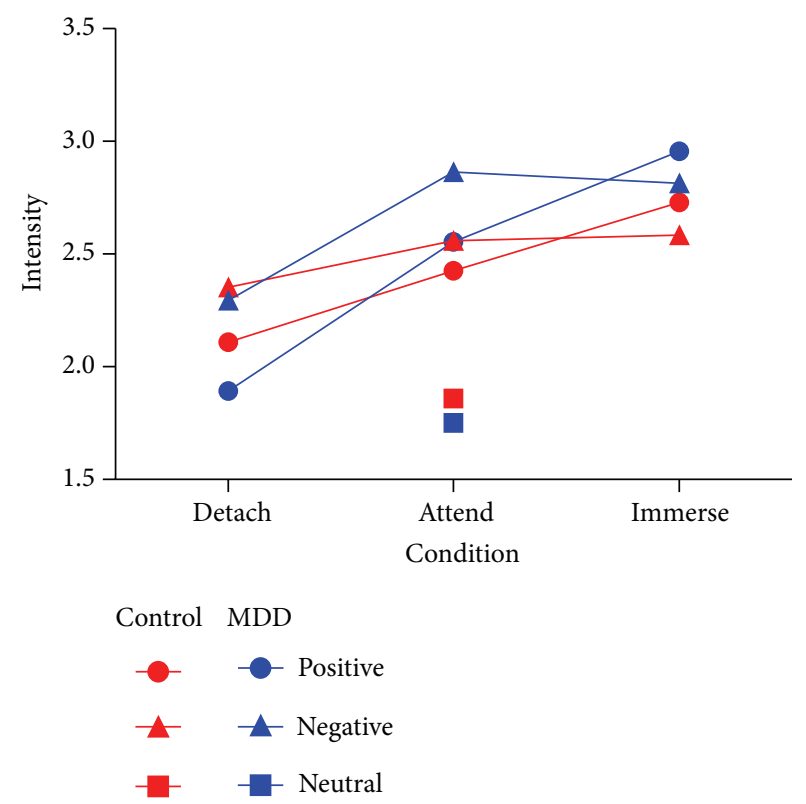

FIGURE 2: Means and standard deviations of intensity ratings after affect processing during the scan.

amygdala coupling were identified with a threshold $P<0.001$ (uncorrected).

\section{Results}

3.1. Behavioral Results. A one-way ANOVA was computed to analyze the effect of picture type (positive, neutral, negative) on induced emotional reactivity during the attending task. A $2 \times 3 \times 2$ repeated-measures ANOVA was also conducted on factors including group (MDD, normal), condition (attend, self-detachment, self-immersion), and emotion (positive, negative) to examine the effects of cognitive reappraisal on emotional induction (Figure 2).

Emotion Reactivity. We observed a significant main effect of emotion $(F(2,22)=44.9, P<0.001)$ during the attending task. Pairwise comparisons showed that negative and positive trials differed from neutral trials $(P<0.001)$. There was no difference between MDD patients and normal controls $(P>$ 0.455).

Emotion Regulation. The emotional state ratings yielded a significant main effect of condition $(F(2,21)=15.620 P=$ 0.000 , partial $\left.\eta^{2}=0.415\right)$ and emotion $(F(1,22)=11.355$, $P=0.003$, partial $\left.\eta^{2}=0.340\right)$ and a significant interaction between condition and emotion $(F(2,21)=14.215, P=0.000$, partial $\left.\eta^{2}=0.575\right)$. Either group main effect or group-related interactions were insignificant $(P>0.05)$. Post hoc contrasts indicated that emotional intensity was significantly regulated via self-detachment $(P=0.001)$ and self-immersion $(P=$ $0.007)$, compared to the viewing condition and to each other $(P=0.001)$. These results suggested that both groups successfully regulated emotions without significant group differences. 


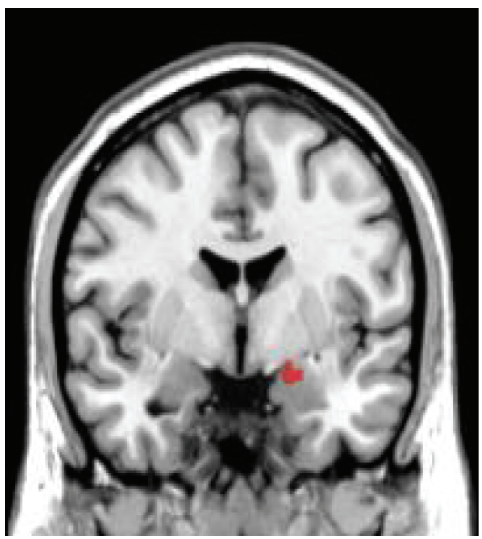

Left amygdala $(-20,-2,-16)$

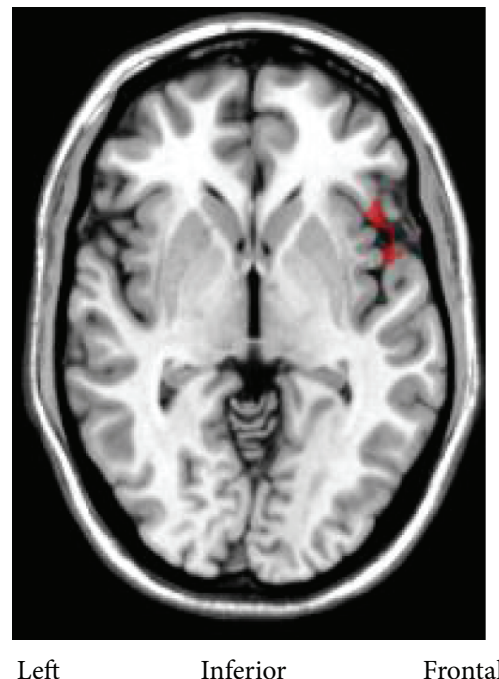

gyrus $(-48,20,-4)$

(a)

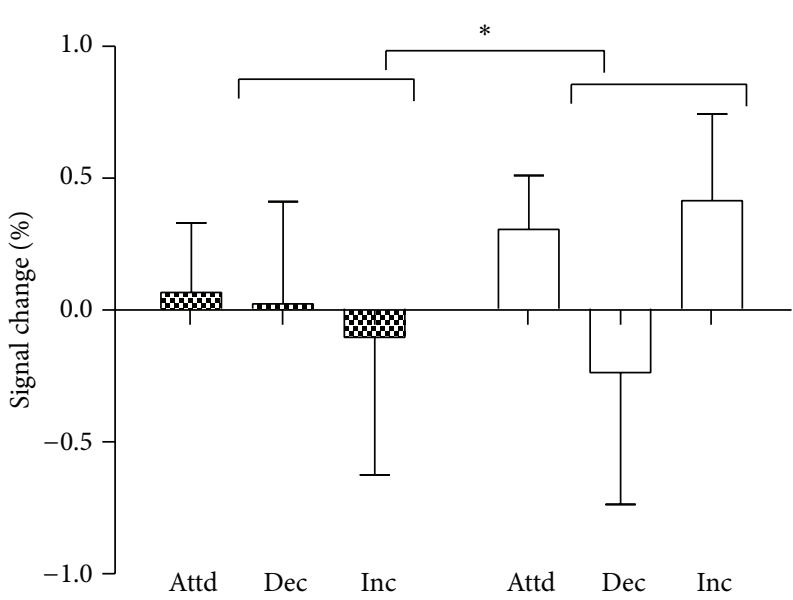

\$2 Depressed Healthy

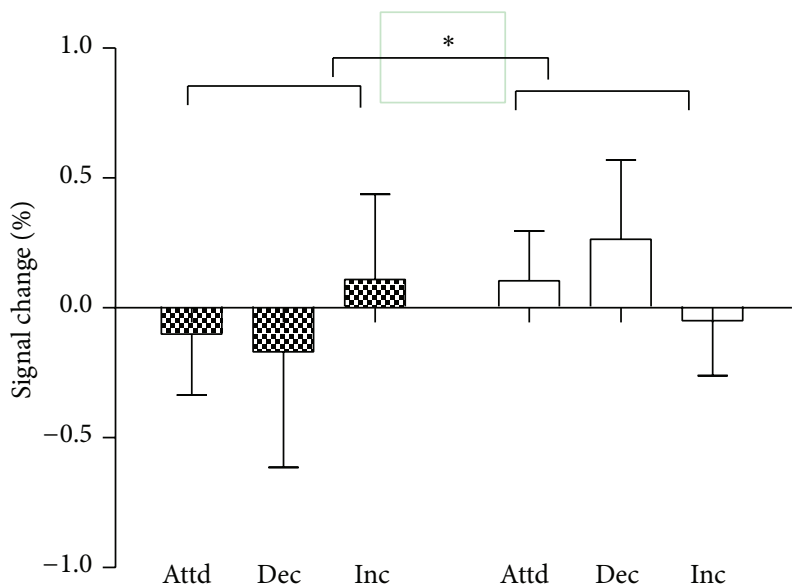

BD Depressed Healthy

Figure 3: (a) Group $\times$ reappraisal interaction: regions activated in "attend," "detach," and "immerse" conditions in both groups. (b) Percent signal change for the following contrasts: "attend/emotional," "detach/emotional," and "immerse/emotional". Note: attd = attend; dec= detach; inc $=$ immerse.

\subsection{Functional MRI Results}

\subsubsection{Activation Analysis}

Factor Analysis. We used a full factorial design ANOVA in the second-level group random-effects analysis. There was no significant group $\times$ reappraisal $\times$ emotion interaction. Group $\times$ reappraisal interactions were found in right medial temporal gyrus (MTG), left inferior frontal gyrus (IFG), left superior temporal gyrus (STG), right lingual gyrus, left thalamus, left amygdala, and left insula.

Regarding priori regions, we found activations in left inferior frontal gyrus (IFG) (109 voxels in left IFG, peak at $(-48,20,-4) t=3.07, P<0.01)$ and left amygdala (40 voxels in left amygdala, peak at $(-20,-2,-16) t=2.42, P<0.01)$.
A post hoc $t$-contrast revealed that the group $\times$ reappraisal interaction was explained by hypoactivation of left amygdala in "immerse minus attend" contrast, hypoactivation of left IFG in "detach minus attend" contrast, and hyperactivation of left IFG in "immerse minus attend" contrast. Percent signal changes in each ROI were extracted and entered into SPSS for group $\times$ reappraisal two-way ANOVA, resulting in similar activation-deactivation pattern (Figure 3). Because we did not observe significant three-way interaction between group, reappraisal, and emotion, we did not take emotional valence into account. We also observed main effects for group, emotion, and reappraisal.

Region of Interest Analysis. To identify the neural correlates of regulatory effects on amygdala activity due to reappraisal, 
TABLE 2: PPI analysis of left amygdala seed for immersion/emotional condition in both groups.

\begin{tabular}{|c|c|c|c|c|c|c|}
\hline \multirow{2}{*}{ Region of coactivation } & \multirow{2}{*}{ Side } & \multirow{2}{*}{$\mathrm{BA}$} & \multicolumn{3}{|c|}{ Talairach coordinates } & \multirow{2}{*}{$Z$ score } \\
\hline & & & $x$ & $y$ & $z$ & \\
\hline \multicolumn{7}{|l|}{ Control > MDD } \\
\hline Middle temporal gyrus & $\mathrm{L}$ & 39 & -52 & -70 & 30 & 2.18 \\
\hline Middle temporal gyrus & $\mathrm{R}$ & 21 & 60 & -18 & -24 & 3.26 \\
\hline Superior temporal gyrus & $\mathrm{R}$ & 38 & 44 & 18 & -34 & 3.05 \\
\hline Medial frontal gyrus & $\mathrm{L}$ & & -12 & 56 & 8 & 2.89 \\
\hline \multicolumn{7}{|l|}{ Control $<$ MDD } \\
\hline Insula & $\mathrm{L}$ & & -40 & -10 & 6 & 5.01 \\
\hline Middle frontal gyrus & $\mathrm{R}$ & 9 & 42 & 42 & 32 & 2.75 \\
\hline Superior frontal gyrus & $\mathrm{R}$ & & 26 & 54 & 32 & 2.46 \\
\hline Inferior parietal lobule & $\mathrm{R}$ & 40 & 42 & -32 & 62 & 2.34 \\
\hline Precuneus & $\mathrm{R}$ & 7 & 18 & -36 & 58 & 2.19 \\
\hline Anterior cingulate & $\mathrm{R}$ & 32 & 10 & 36 & -10 & 2.16 \\
\hline Precuneus & $\mathrm{L}$ & & -6 & -44 & 50 & 1.97 \\
\hline
\end{tabular}

we performed ROI analysis on bilateral amygdala using onesample $t$ test in the control group. For detach effects, we observed decreased amygdala activity during "attend/positive $>$ detach/positive" contrast ( 9 voxels in left amygdala, peak at $(-18,-4,-18) t=2.17, P<0.05)$ and decreased amygdala activity during "attend/negative $>$ detach/negative" contrast (6 voxels in left amygdala, peak at $(-20,-10,-10) t=2.57, P<$ $0.05)$. For immerse effects, we observed increased amygdala activity during "immerse/positive $>$ attend/positive" contrast (30 voxels in right amygdala, peak at $(22,-6,-18) t=3.56$, $P<0.005)$ and during "immerse/negative $>$ attend/negative" contrast (11 voxels in left amygdala, peak at $(-18,-4,-26)$ $t=2.77, P<0.01 ; 44$ voxels in right amygdala, peak at $(20$, $-4,-26) t=4.57, P<0.001)$. We performed similar test on the patient group and found no regulatory effects of amygdala in any individual contrast.

We then performed a two-sample $t$ test for anatomical ROIs between MDD and the control group. The investigation of all contrasts of interest did not include bilateral amygdala, as no regulation effects of amygdala were found in patients and thus incomparable between groups. For "detach/positive $>$ attend/positive" condition, left vmPFC showed greater activation for patients than for controls (15 voxels in left vmPFC, peak at $(-4,54,-10) t=2.61, P<0.01)$. For "detach/negative $>$ attend/negative" condition, right dlPFC and vmPFC were more active in patients than in controls (8 voxels in right dlPFC, peak at $(14,40,20) t=1.99, P<0.05$; 37 voxels in right vmPFC, peak at $(38,34,-14) t=3.71$, $P<0.001 ; 18$ voxels in right vmPFC, peak at $(6,52,-10)$ $t=3.13, P<0.005$; 53 voxels in right vmPFC, peak at $(10$, $34,20) t=2.22, P<0.05)$. For "immerse/positive $>$ attend/ positive" condition, left vmPFC was more active in patients than in controls, and right vmPFC was more active in controls than in patients $(20$ voxels in left vmPFC, peak at $(-4,56$, -6) $t=2.23, P<0.05 ; 7$ voxels in right vmPFC, peak at $(24,34,-12) t=0.02, P<0.05)$. For “immerse/negative $>$ attend/negative" condition, right dlPFC and bilateral vmPFC showed greater activation for patients than for controls (12 voxels in right dlPFC, peak at $(12,40,18) t=2.26, P<0.05$;
12 voxels in left vmPFC, peak at $(-4,46,12) t=1.84, P<0.05$; 66 voxels in right vmPFC, peak at $(10,38,18) t=2.52$, $P<0.01)$.

In patients, a regression analysis revealed that during detachment of positive emotion, downregulation of left amygdala negatively correlated with HAMD scores $(r=$ $-0.608, P=0.036$, two-tailed), suggesting that the more severe the depression symptom is, the less effective the downregulation of amygdala will be (Figure 4).

3.2.2. Psychophysiological Interaction Analysis. We are specifically interested in amygdala-cortical interactions during reappraisal. The PPI analysis revealed that compared to healthy controls, patients showed significantly enhanced coactivation of left amygdala with right dlPFC (MFG), right vmPFC (anterior cingulate), and right inferior parietal lobule (IPL) (Table 2).

\section{Discussions}

The current study extends previous findings about neural correlates of reappraisal in MDD along a self-relatedness dimension and confirms the hypotheses that self-relatedness may differentially modulate neural circuits underlying reappraisal for MDD versus normal group, demonstrating inflexible amygdala reactivity and strengthening frontolimbic connection in MDD. Since these neural circuits are involved in the pathology of depression [37], the current study may provide further evidence on how this abnormal functional connectivity pattern translates into emotion dysregulation in depression.

Behaviorally, IAPS stimuli significantly induced emotion in both groups $(P<0.05)$. In addition, both groups were equally effective in using reappraisal strategies to up- and downregulate emotions. Neurally, within-group region-ofinterest analysis indicates the regulation effects of reappraisal on amygdala in controls, consistent with previous studies [38-40], suggesting neural correlates as more sensitive 


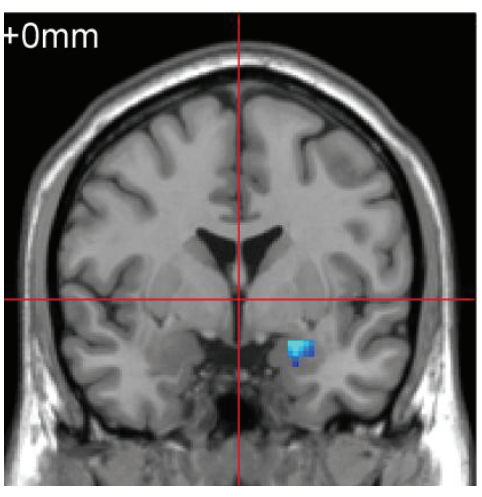

(a)

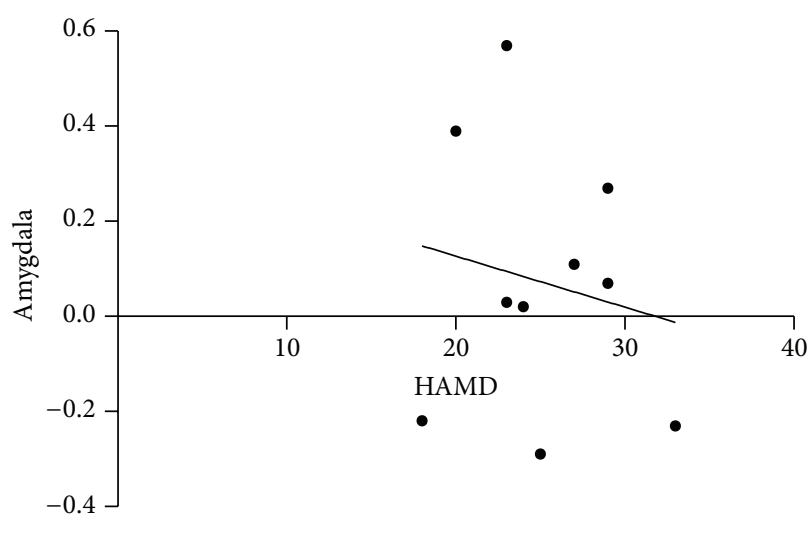

(b)

FIGURE 4: (a) Decreased activation in the left amygdala for patients during detachment of positive emotion. (b) The signal change of amygdala between the detachment and view conditions correlated negatively with HAMD scores $(r=-0.608, P=0.036)$.

indices of regulation outcome. It is noteworthy that this pattern of amygdala reactivity was not observed in the MDD group. Previous studies are inconclusive regarding whether the ability to regulate amygdala activation is deteriorated in MDD. One possible explanation for the discrepancy between studies is the heterogeneity of regulation goals, which use self-ratings of valence and/or arousal as behavioral measures. Some studies report that controls and depressed individuals show comparable amygdala responses to emotional stimuli in "detach $>$ attend" contrast $[6,12,41]$. However, Johnstone's study does not observe "decrease-attend" reappraisal effect on amygdala activation in either controls or depressed patients [13]. Empirical evidence indicates that amygdala belongs to both valence and arousal networks [42]. In particular, amygdala is sensitive to arousal when valence remains unchanged and is dormant to valence changes when arousal remains constant [43]. Thus, regulation goals putatively cause distinct amygdala response patterns which may be ignored in previous studies.

Interestingly, Dillon's study using valence ratings as emotion responses revealed comparable regulation outcome between groups in increasing emotion, which differs from current study. Moreover, post hoc contrasts of repeatedmeasures ANOVA demonstrate that the left amygdala of depressed patients is less activated when immersion strategy is adopted regardless of valence (see in Figure 3). This blunted amygdala activity is aligned with an emotion context insensitivity (ECI) view which depicted flattened emotional responses typical of MDD [44]. Accordingly, event-related potential (ERP) study also addressed diminished brain responses during sustained processing of positive information [45]. Our study was comparable to previous study showing that depression fails to maintain positive emotions, with a different brain foci of positive emotion processing (ventral striatum) [19]. The disparity between studies is comprehensible in that our study adopted emotional arousal as an indicator of regulation outcome, while in Heller's study, emotional valence was adopted. Furthermore, less amygdala activity during immersion is also consistent with its role in representation with arousal [46].
In contrast to healthy controls, depressed individuals exhibit diminished activation in left IFG when detachment strategy is adopted and enhanced activation in left IFG when immersion technique is adopted (see in Figure 3). IFG (BA47) is implicated in inhibitory control in emotional as well as cognitive domains [47]. This abnormal control-related activation can be viewed as a functionally compensatory process in response to behavioral deficits, in spite of preserved emotion regulation behavioral measures for MDD. Altogether, the present results indicate compromised functioning of MDD in resistance to affective interference and inhibiting spontaneous emotional responses and supported the assumption that MDD emotion dysregulation is spanning negative as well as positive affect.

Our study observes that in depressed patients, left vmPFC is more strongly recruited for self-detachment from positive affect, while right vmPFC is more strongly activated for negative affect. Observations are similar concerning selfimmersion strategy. Medial prefrontal cortex (MPFC) is central to neural models of depression [48]. In resting state, MDD patients exhibit overall increase in ventromedial PFC activation from pre- to posttreatment [49]. Task-related vmPFC activation is observed in self-oriented reappraisal [21] or when negative emotion is decreased [50]. VmPFC may be responsible for preattentively tagging both explicit and implicit incoming information as self-relevant [51, 52], representing the "Me" mode of self-reflection [53]. Our observations suggest that under both self-detached and selfimmersed conditions, depressed patients show an excessive mode of self-relevance detection. This may in turn orient ongoing dorsal-ventral PFC connectivity, in line with positive correlation of elevations of negative affect with brain activations in medial PFC [54]. This self-focused cognitive tendency may constitute a basis for rumination style preferentially prevalent in depression [55].

We further assume that comparisons within each valence might suggest a possibility for hemispheric asymmetry of prefrontal cortex in MDD: left vmPFC is more involved in positive affect reappraisal and right vmPFC more in negative affect reappraisal. However, more conclusive results 
may rely on ANOVA including "hemisphere" as one of the factors, which is not considered in this paper because SPM 8 does not allow four-way ANOVA. It is in line with the consensus that positive/negative emotion is parallel to approach/avoidance motivation system [56], which shows evidence of hemispheric specialization in MFG [57].

The PPI analysis exhibited a strengthened coupling between left amygdala and right prefrontal cortex (including dlPFC and vmPFC) and right IPL in MDD when increasing emotion. In comparison, two-sample $t$ test of whole brain analysis showed enhanced activations of dlPFC and vmPFC in MDD, together with over-reactivity of limbic-paralimbic (insula, parahippocampal gyrus) and subcortical (thalamus) structures. DIPFC (BA9, 46) is seen as the neural substrate for emotion regulation [58] and is more preferentially engaged in negative than positive stimuli in depressed patients [59]. Furthermore, dlPFC is responsible for recruiting attention control resources in reappraisal [60] and is more engaged with increasing cognitive load [61]. This result further supports our proposal that MDD may have deficits in upregulating emotion accompanied with heightened inhibitory control. Right IPL is involved in downregulation of emotion through detachment in healthy and depressed group [12], while the absence of detachment-related coactivation with amygdala for MDD is not discussed. Our findings further address a strengthened coupling of right IPL and amygdala for MDD during enhancing emotion. IPL is associated with cognitive inhibition [62], taking the perspectives of others in processing visual information [63] or orienting away from salient stimuli [64]. Collectively, from detachment to immersion, impaired functional connectivity between IPL and amygdala emphasize that depressed patients may have deficits in reappraisal which is essential for regulating emotion in both directions.

A wealth of data suggests that amygdala, insula, and anterior temporal pole are responsible for separately mediating the cognitive, physiological, and experiential aspects of emotional responses, respectively [41]. The insula serves as a strategic neural node in the appraisal of emotional responses [65]. We observed strengthened task-related coupling of amygdala with insula; amygdala response under self-immersion condition was less active in patients than in controls, implying the attenuated ability of MDD to flexibly intensify emotion reactivity. This also confirms MDD's positive emotion dysregulation assumption.

In conclusion, depressed individuals tend to rely more on cognitive control brain networks and enhanced functional coupling between left amygdala and right prefrontal cortex when using reappraisal strategy accompanied by unrestrained self-related affective processing, which applies for both valence of emotion.

4.1. Implications and Limitations. There may be several clinical implications of our findings. First, group differences in the ability to regulate emotion may represent a sign of vulnerability to depressed mood and depressive disorders under stress [66]. Thus, focusing on affect regulation provides a ready bridge to intervention research [67]. Second, the abnormal prefrontal activation in response to an emotion-eliciting context may be second, since the amount of downregulation of positive emotion changes with depression severity, which does not necessarily disappear with symptom recovery or medication. The underlying neurobiological changes could be used to monitor the responsiveness of patients and the effectiveness of psychotherapy.

Until now, the effect of cross-culture variability on reappraisal strategies remains largely unknown. Our study may provide preliminary insights into relevant research. The linguistic nature of emotion regulation strategies may vary among different cultures. As previous findings suggest, emotion-regulation strategies may contribute to differences in emotional experience across western and east Asian cultures [68]. Hence, replication and comparative study between ethnic groups should be targeted.

The present research has several limitations. First, stimuli of personal relevance may need to be adopted (such as autobiographical experiences) in further study. Reappraisal involves momentary relevance and meaning of current stimuli which may vary among individuals, and subject-specific stimuli according to personal relevance may maintain stimulus consistency within individuals [69]. Second, this study does not allow making causal conclusions of reciprocal connection between brain regions. This issue could be further investigated with methods such as dynamic causal modeling (DCM) or granger causal modeling (GCM) for more confirmative conclusions about causal relations or in combination with ERP technique to keep track of ongoing mental processes on finer time scale. Third, the sample size of this study is small, but the findings are well aligned with previous studies of emotion regulation. The findings in this study can serve as a basis for further investigation with a larger sample size and stronger statistical power [70].

\section{Abbreviations \\ dlPFC: Dorsal lateral prefrontal cortex \\ IFG: Inferior frontal gyrus \\ vmPFC: Ventral medial prefrontal cortex \\ DSM-IV: Fourth edition of Diagnostic and Statistical Manual of Mental Disorders \\ MDD: $\quad$ Major depressive disorder \\ CBT: Cognitive behavioral therapy \\ BDI: Beck depression inventory \\ SDS: $\quad$ Self-rating depression scale \\ HAMD: Hamilton rating scale for depression \\ IAPS: International affective pictures system \\ FOV: $\quad$ Field of view \\ TE: $\quad$ Time echo \\ TR: $\quad$ Time repetition \\ ROI: $\quad$ Region of interests \\ VOI: Volume of interests \\ PPI: Psychophysiological interaction.}

\section{Conflict of Interests}

The authors declare that there is no conflict of interests regarding the publication of this paper. 


\section{Acknowledgments}

This research was financially supported by National Natural Science Foundation of China (NSFC30970898). The authors gratefully acknowledge the valuable assistance of Professor Huaqing Meng (the First Affiliated Hospital of Chongqing Medical University, China) and Dr. Chenggang Jiang (Institute of Surgery Research of Daping Hospital, China) for patient recruitment, Dr. Jiuquan Zhang for professional advice of imaging data processing, and Chengju Liao, Jiawen Li, Yun Liu, and Liying Gan for helpful comments.

\section{References}

[1] A. Aldao, S. Nolen-Hoeksema, and S. Schweizer, "Emotion-regulation strategies across psychopathology: a meta-analytic review," Clinical Psychology Review, vol. 30, no. 2, pp. 217-237, 2010.

[2] H. Lee, A. S. Heller, C. M. van Reekum, B. Nelson, and R. J. Davidson, "Amygdala-prefrontal coupling underlies individual differences in emotion regulation," Neuroimage, vol. 62, no. 3 , pp. 1575-1581, 2012.

[3] J.-A. Min, J. J. Yu, C. U. Lee, and J.-H. Chae, "Cognitive emotion regulation strategies contributing to resilience in patients with depression and/or anxiety disorders," Comprehensive Psychiatry, vol. 54, no. 8, pp. 1190-1197, 2013.

[4] S. G. Disner, C. G. Beevers, E. A. P. Haigh, and A. T. Beck, "Neural mechanisms of the cognitive model of depression," Nature Reviews: Neuroscience, vol. 12, no. 8, pp. 467-477, 2011.

[5] K. J. Ressler and H. S. Mayberg, "Targeting abnormal neural circuits in mood and anxiety disorders: from the laboratory to the clinic," Nature Neuroscience, vol. 10, no. 9, pp. 1116-1124, 2007.

[6] D. G. Dillon and D. A. Pizzagalli, "Evidence of successful modulation of brain activation and subjective experience during reappraisal of negative emotion in unmedicated depression," Psychiatry Research: Neuroimaging, vol. 212, no. 2, pp. 99-107, 2013.

[7] P. Kanske, J. Heissler, S. Schönfelder, and M. Wessa, "Neural correlates of emotion regulation deficits in remitted depression: the influence of regulation strategy, habitual regulation use, and emotional valence," NeuroImage, vol. 61, no. 3, pp. 686-693, 2012.

[8] G. Perlman, A. N. Simmons, J. Wu et al., "Amygdala response and functional connectivity during emotion regulation: a study of 14 depressed adolescents," Journal of Affective Disorders, vol. 139, no. 1, pp. 75-84, 2012.

[9] M. J. Smoski, S. L. Keng, C. E. Schiller, J. Minkel, and G. S. Dichter, "Neural mechanisms of cognitive reappraisal in remitted major depressive disorder," Journal of Affective Disorders, vol. 151, no. 1, pp. 171-177, 2013.

[10] J. T. Buhle, J. A. Silvers, T. D. Wager et al., "Cognitive reappraisal of emotion: a meta-analysis of human neuroimaging studies," Cereb Cortex, 2013.

[11] N. Garnefski and V. Kraaij, "The cognitive emotion regulation questionnaire: psychometric features and prospective relationships with depression and anxiety in adults," European Journal of Psychological Assessment, vol. 23, no. 3, pp. 141-149, 2007.

[12] S. Erk, A. Mikschl, S. Stier et al., "Acute and sustained effects of cognitive emotion regulation in major depression," Journal of Neuroscience, vol. 30, no. 47, pp. 15726-15734, 2010.
[13] T. Johnstone, C. M. van Reekum, H. L. Urry, N. H. Kalin, and R. J. Davidson, "Failure to regulate: counterproductive recruitment of top-down prefrontal-subcortical circuitry in major depression," Journal of Neuroscience, vol. 27, no. 33, pp. 8877-8884, 2007.

[14] K. N. Ochsner and J. J. Gross, “Thinking makes it so: a social cognitive neuroscience approach to emotion regulation," in Handbook of Self-Regulation: Research, Theory, and Applications, R. F. Baumeister and K. D. Vohs, Eds., pp. 229-255, 2004.

[15] K. McRae, B. Ciesielski, and J. J. Gross, "Unpacking cognitive reappraisal: goals, tactics, and outcomes," Emotion, vol. 12, no. 2, pp. 250-255, 2012.

[16] A. K. Y. Mak, Z.-G. Hu, J. X. Zhang, Z.-W. Xiao, and T. M. C. Lee, "Neural correlates of regulation of positive and negative emotions: an fMRI study," Neuroscience Letters, vol. 457, no. 2, pp. 101-106, 2009.

[17] M. M. Tugade and B. L. Fredrickson, "Resilient individuals use positive emotions to bounce back from negative emotional experiences," Journal of Personality and Social Psychology, vol. 86, no. 2, pp. 320-333, 2004.

[18] E. L. Garland, B. Fredrickson, A. M. Kring, D. P. Johnson, P. S. Meyer, and D. L. Penn, "Upward spirals of positive emotions counter downward spirals of negativity: insights from the broaden-and-build theory and affective neuroscience on the treatment of emotion dysfunctions and deficits in psychopathology," Clinical Psychology Review, vol. 30, no. 7, pp. 849-864, 2010.

[19] A. S. Heller, T. Johnstone, A. J. Shackman et al., "Reduced capacity to sustain positive emotion in major depression reflects diminished maintenance of fronto-striatal brain activation," Proceedings of the National Academy of Sciences of the United States of America, vol. 106, no. 52, pp. 22445-22450, 2009.

[20] F. A. Huppert, "Positive emotions and cognition developmental, neuroscience and health perspectives," in Proceedings of the 8th Sydney Symposium, 2005.

[21] K. N. Ochsner, R. D. Ray, J. C. Cooper, E. R. Robertson, S. Chopra, J. D. E. Gabrieli et al., "For better or for worse: neural systems supporting the cognitive down- and up-regulation of negative emotion,” NeuroImage, vol. 53, no. 1, pp. 11-20, 2004.

[22] R. D. Ray and D. H. Zald, "Anatomical insights into the interaction of emotion and cognition in the prefrontal cortex," Neuroscience and Biobehavioral Reviews, vol. 36, no. 1, pp. 479501, 2012.

[23] I. A. Cristea, A. S. Tatar, D. Nagy, and D. David, "The bottle is half empty and that's bad, but not tragic: differential effects of negative functional reappraisal," Motivation and Emotion, vol. 36, no. 4, pp. 550-563, 2012.

[24] M. N. Shiota and R. W. Levenson, "Effects of aging on experimentally instructed detached reappraisal, positive reappraisal, and emotional behavior suppression," Psychology and Aging, vol. 24, no. 4, pp. 890-900, 2009.

[25] M. N. Shiota and R. W. Levenson, "Turn down the volume or change the channel? Emotional effects of detached versus positive reappraisal," Journal of Personality and Social Psychology, vol. 103, no. 3, pp. 416-429, 2012.

[26] E. Kross and Ö. Ayduk, "Boundary conditions and buffering effects: does depressive symptomology moderate the effectiveness of self-distancing for facilitating adaptive emotional analysis?" Journal of Research in Personality, vol. 43, no. 5, pp. 923-927, 2009. 
[27] E. Kross and O. Ayduk, "Making meaning out of negative experiences by self-distancing," Current Directions in Psychological Science, vol. 20, no. 3, pp. 187-191, 2011.

[28] E. Kross, D. Gard, P. Deldin, J. Clifton, and O. Ayduk, “'Asking why" from a distance: its cognitive and emotional consequences for people with major depressive disorder," Journal of Abnormal Psychology, vol. 121, no. 3, pp. 559-569, 2012.

[29] Y.-L. Wang, Y.-C. Lin, C.-L. Huang, and K.-H. Yeh, "Benefitting from a different perspective: the effect of a complementary matching of psychological distance and habitual perspective on emotion regulation," Asian Journal of Social Psychology, vol. 15, no. 3, pp. 198-207, 2012.

[30] C. Lemogne, H. Mayberg, L. Bergouignan et al., "Self-referential processing and the prefrontal cortex over the course of depression: a pilot study," Journal of Affective Disorders, vol. 124, no. 1-2, pp. 196-201, 2010.

[31] S. Grimm, C. F. Schmidt, F. Bermpohl et al., "Segregated neural representation of distinct emotion dimensions in the prefrontal cortex-an fMRI study," NeuroImage, vol. 30, no. 1, pp. 325-340, 2006.

[32] P. J. Lang, M. M. Bradley, and B. N. Cuthbert, International Affective Picture System (IAPS): Affective Ratings of Pictures and Instruction Manual, University of Florida, Gainesville, Fla, USA, 2008.

[33] P. Vrtička, D. Sander, and P. Vuilleumier, "Effects of emotion regulation strategy on brain responses to the valence and social content of visual scenes," Neuropsychologia, vol. 49, no. 5, pp. 1067-1082, 2011.

[34] K. McRae, K. N. Ochsner, I. B. Mauss, J. J. D. Gabrieli, and J. J. Gross, "Gender differences in emotion regulation: an fMRI study of cognitive reappraisal," Group Processes \& Intergroup Relations, vol. 11, no. 2, pp. 143-162, 2008.

[35] S. J. Banks, K. T. Eddy, M. Angstadt, P. J. Nathan, and K. L. Phan, "Amygdala-frontal connectivity during emotion regulation," Social Cognitive and Affective Neuroscience, vol. 2, no. 4, pp. 303312, 2007.

[36] E. P. Duff, R. Cunnington, and G. F. Egan, "REX: response exploration for neuroimaging datasets," Neuroinformatics, vol. 5, no. 4, pp. 223-234, 2007.

[37] Y. Liao, X. Huang, Q. Wu, C. Yang, W. Kuang, and M. Du, "Is depression a disconnection syndrome? Meta-analysis of diffusion tensor imaging studies in patients with MDD," Journal of Psychiatry \& Neuroscience, vol. 38, no. 1, pp. 49-56, 2013.

[38] N. R. Giuliani, K. McRae, and J. J. Gross, “The up- and downregulation of amusement: experiential, behavioral, and autonomic consequences," Emotion, vol. 8, no. 5, pp. 714-719, 2008.

[39] H. K. Sang and S. Hamann, "Neural correlates of positive and negative emotion regulation," Journal of Cognitive Neuroscience, vol. 19, no. 5, pp. 776-798, 2007.

[40] J. Lévesque, F. Eugène, Y. Joanette et al., "Neural circuitry underlying voluntary suppression of sadness," Biological Psychiatry, vol. 53, no. 6, pp. 502-510, 2003.

[41] M. Beauregard, V. Paquette, and J. Levesque, "Dysfunction in the neural circuitry of emotional self-regulation in major depressive disorder," Neuroreport, vol. 17, no. 8, pp. 843-846, 2006.

[42] L. B. Baucom, D. H. Wedell, J. Wang, D. N. Blitzer, and S. V. Shinkareva, "Decoding the neural representation of affective states," NeuroImage, vol. 59, no. 1, pp. 718-727, 2012.

[43] A. K. Anderson and N. Sobel, "Dissociating intensity from valence as sensory inputs to emotion," Neuron, vol. 39 , no. 4 , pp. 581-583, 2003.
[44] L. M. Bylsma, B. H. Morris, and J. Rottenberg, "A meta-analysis of emotional reactivity in major depressive disorder," Clinical Psychology Review, vol. 28, no. 4, pp. 676-691, 2008.

[45] A. Y. Shestyuk, P. J. Deldin, J. E. Brand, and C. M. Deveney, "Reduced sustained brain activity during processing of positive emotional stimuli in major depression," Biological Psychiatry, vol. 57, no. 10, pp. 1089-1096, 2005.

[46] F. C. Murphy, I. Nimmo-Smith, and A. D. Lawrence, "Functional neuroanatomy of emotions: a meta-analysis," Cognitive, Affective and Behavioral Neuroscience, vol. 3, no. 3, pp. 207-233, 2003.

[47] D. E. Payer, K. Baicy, M. D. Lieberman, and E. D. London, "Overlapping neural substrates between intentional and incidental down-regulation of negative emotions," Emotion, vol. 12, no. 2, pp. 229-235, 2012.

[48] C. Lemogne, P. Delaveau, M. Freton, S. Guionnet, and P. Fossati, "Medial prefrontal cortex and the self in major depression," Journal of Affective Disorders, vol. 136, no. 1-2, pp. el-el1, 2012.

[49] M. Ritchey, F. Dolcos, K. M. Eddington, T. J. Strauman, and R. Cabeza, "Neural correlates of emotional processing in depression: changes with cognitive behavioral therapy and predictors of treatment response," Journal of Psychiatric Research, vol. 45, no. 5, pp. 577-587, 2011.

[50] H. L. Urry, C. M. van Reekum, T. Johnstone et al., "Amygdala and ventromedial prefrontal cortex are inversely coupled during regulation of negative affect and predict the diurnal pattern of cortisol secretion among older adults," Journal of Neuroscience, vol. 26, no. 16, pp. 4415-4425, 2006.

[51] L. T. Rameson, A. B. Satpute, and M. D. Lieberman, "The neural correlates of implicit and explicit self-relevant processing," NeuroImage, vol. 50, no. 2, pp. 701-708, 2010.

[52] T. W. Schmitz and S. C. Johnson, "Relevance to self: a brief review and framework of neural systems underlying appraisal," Neuroscience and Biobehavioral Reviews, vol. 31, no. 4, pp. 585596, 2007.

[53] K. N. Ochsner and J. J. Gross, "Putting the "I" and the "Me" in emotion regulation: reply to Northoff," Trends in Cognitive Sciences, vol. 22, no. 2, pp. 126-131, 2005.

[54] C. S. Carter, "The ups and downs of emotion regulation," Biological Psychiatry, vol. 65, no. 5, pp. 359-360, 2009.

[55] J. Roelofs, L. Rood, C. Meesters et al., "The influence of rumination and distraction on depressed and anxious mood: a prospective examination of the response styles theory in children and adolescents," European Child and Adolescent Psychiatry, vol. 18, no. 10, pp. 635-642, 2009.

[56] M. Mneimne, A. S. Powers, K. E. Walton, D. S. Kosson, S. Fonda, and J. Simonetti, "Emotional valence and arousal effects on memory and hemispheric asymmetries," Brain and Cognition, vol. 74, no. 1, pp. 10-17, 2010.

[57] J. M. Spielberg, G. A. Miller, A. S. Engels et al., “Trait approach and avoidance motivation: lateralized neural activity associated with executive function," NeuroImage, vol. 54, no. 1, pp. 661-670, 2011.

[58] K. N. Ochsner, S. A. Bunge, J. J. Gross, and J. D. E. Gabrieli, "Rethinking feelings: an fMRI study of the cognitive regulation of emotion," Journal of Cognitive Neuroscience, vol. 14, no. 8, pp. 1215-1229, 2002.

[59] B.-T. Lee, S. W. Cho, H. S. Khang et al., "The neural substrates of affective processing toward positive and negative affective pictures in patients with major depressive disorder," Progress in Neuro-Psychopharmacology and Biological Psychiatry, vol. 31, no. 7, pp. 1487-1492, 2007. 
[60] K. McRae, B. Hughes, S. Chopra, J. D. E. Gabrieli, J. J. Gross, and K. N. Ochsner, "The neural bases of distraction and reappraisal," Journal of Cognitive Neuroscience, vol. 22, no. 2, pp. 248-262, 2010.

[61] L. F. van Dillen, D. J. Heslenfeld, and S. L. Koole, “Tuning down the emotional brain: an fMRI study of the effects of cognitive load on the processing of affective images," NeuroImage, vol. 45, no. 4, pp. 1212-1219, 2009.

[62] S. L. Warren, L. D. Crocker, J. M. Spielberg, A. S. Engels, M. T. Banich, B. P. Sutton et al., "Cortical organization of inhibitionrelated functions and modulation by psychopathology," Frontiers in Human Neuroscience, vol. 7, article 271, 2013.

[63] H. Shibata and T. Inui, "Brain activity associated with recognition of appropriate action selection based on allocentric perspectives," Neuroscience Letters, vol. 491, no. 3, pp. 187-191, 2011.

[64] B. E. Benson, M. W. Willis, T. A. Ketter, A. Speer, T. A. Kimbrell, and M. S. George, "Interregional cerebral metabolic associativity during a continuous performance task (part II): differential alterations in bipolar and unipolar disorders," Psychiatry Research: Neuroimaging, vol. 164, no. 1, pp. 30-47, 2008.

[65] R. Veit, V. Singh, R. Sitaram, A. Caria, K. Rauss, and N. Birbaumer, "Using real-time fMRI to learn voluntary regulation of the anterior insula in the presence of threat-related stimuli," Social Cognitive and Affective Neuroscience, vol. 7, no. 6, pp. 623634, 2012.

[66] C. A. Burghy, D. E. Stodola, P. L. Ruttle, E. K. Molloy, J. M. Armstrong, and J. A. Oler, "Developmental pathways to amygdala-prefrontal function and internalizing symptoms in adolescence," Nature Neuroscience, vol. 15, no. 12, pp. 1736-1741, 2012.

[67] M. Kovacs, J. Joormann, and I. H. Gotlib, "Emotion (Dys) regulation and links to depressive disorders," Child Development Perspectives, vol. 2, no. 3, pp. 149-155, 2008.

[68] E. Davis, E. Greenberger, S. Charles, C. Chen, L. Zhao, and Q. Dong, "Emotion experience and regulation in China and the United States: how do culture and gender shape emotion responding?" International Journal of Psychology, vol. 47, no. 3, pp. 230-239, 2012.

[69] J. J. Campos, E. A. Walle, A. Dahl, and A. Main, "Reconceptualizing emotion regulation," Emotion Review, vol. 3, no. 1, pp. 26-35, 2011.

[70] A. K. Y. Mak, M. M. C. Wong, S.-H. Han, and T. M. C. Lee, "Gray matter reduction associated with emotion regulation in female outpatients with major depressive disorder: a voxel-based morphometry study," Progress in Neuro-Psychopharmacology and Biological Psychiatry, vol. 33, no. 7, pp. 1184-1190, 2009. 

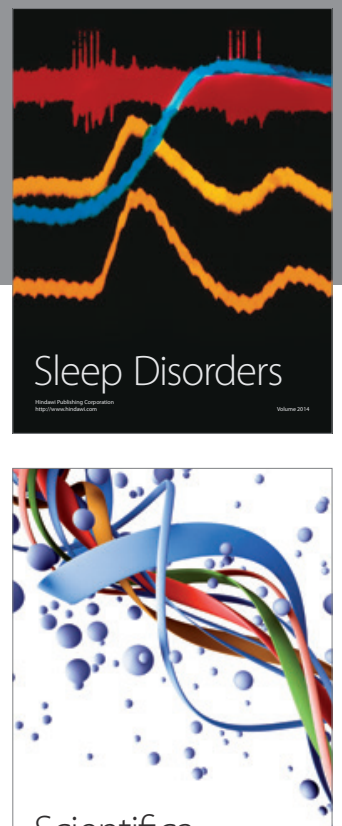

Scientifica
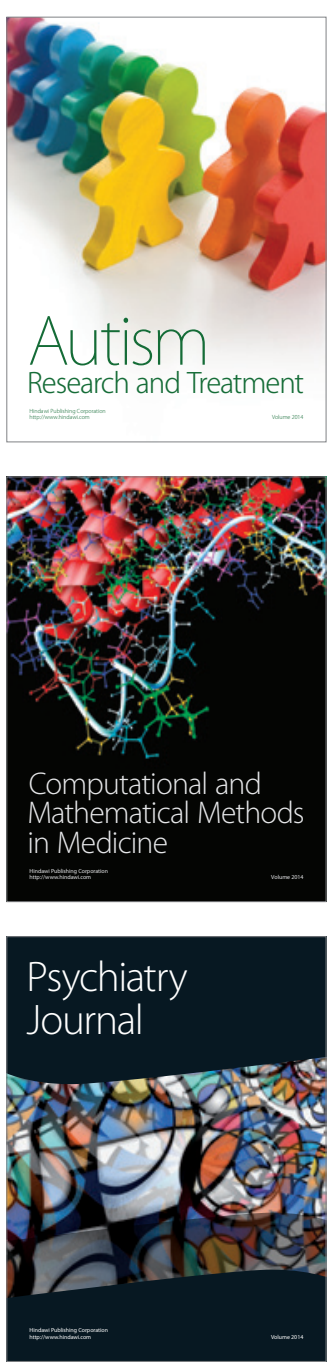
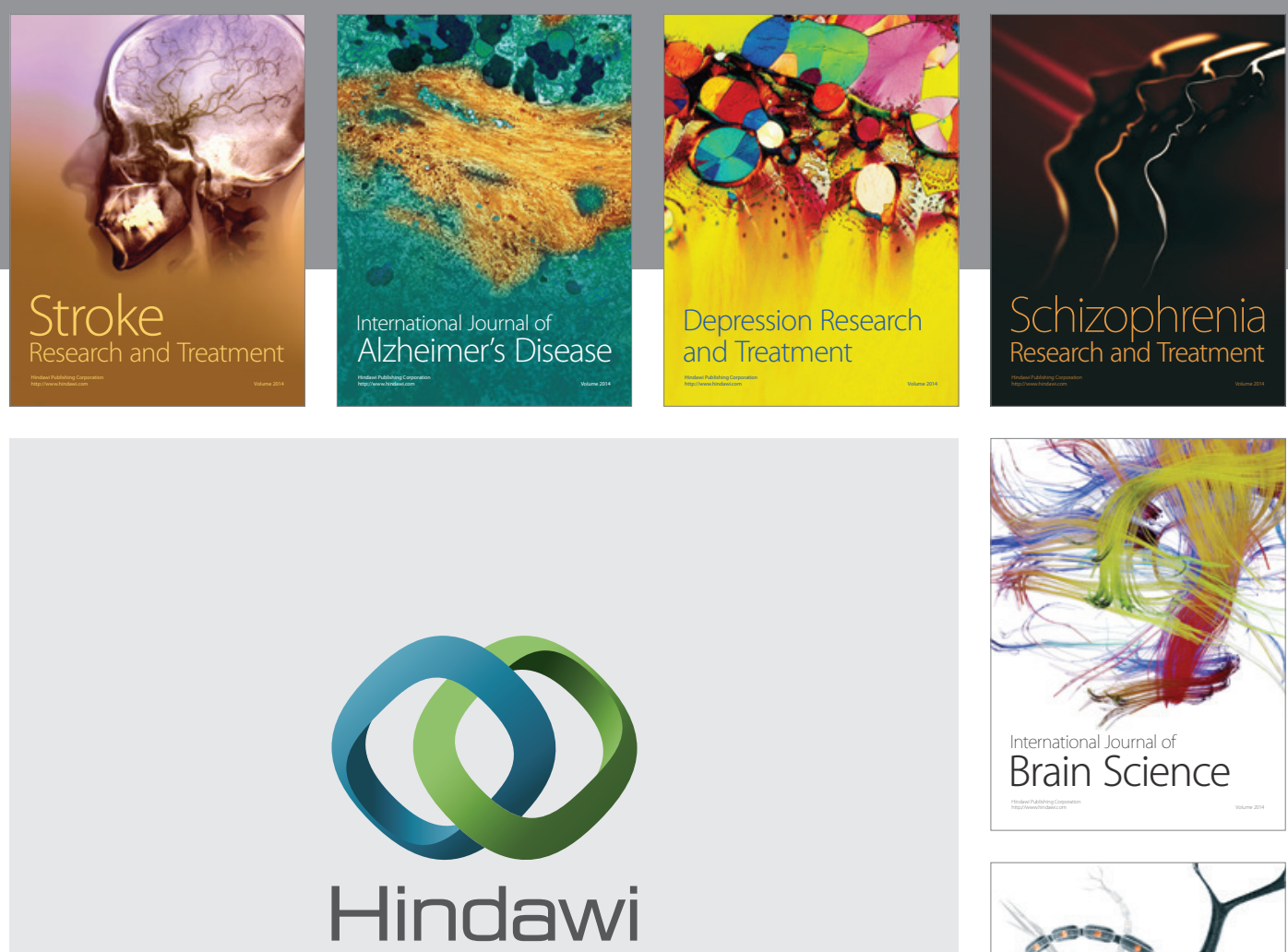

Submit your manuscripts at

http://www.hindawi.com
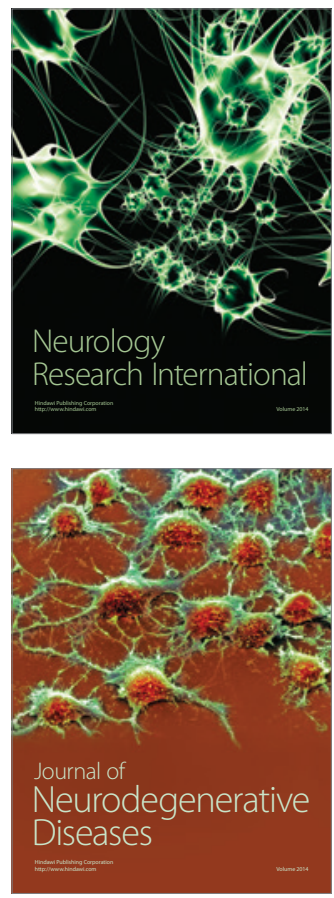

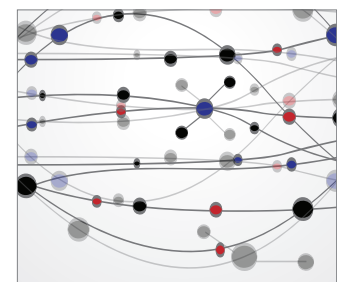

The Scientific World Journal
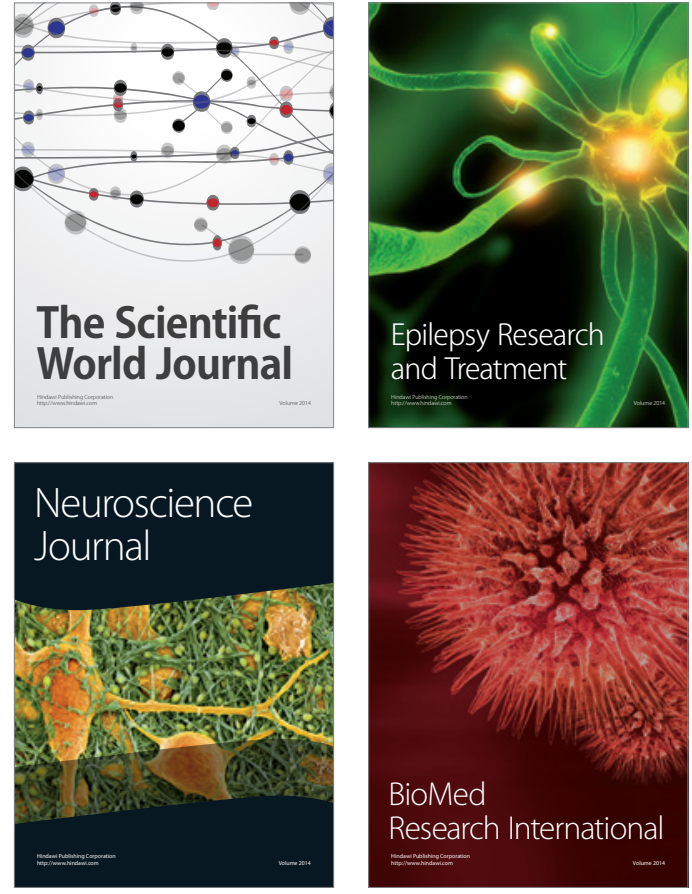

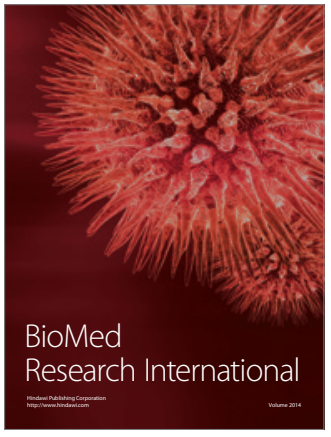

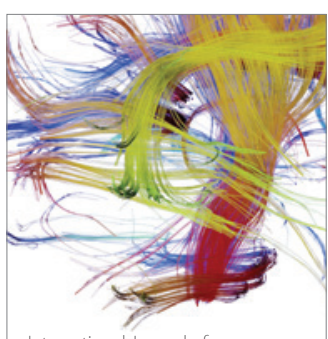

Brain Science

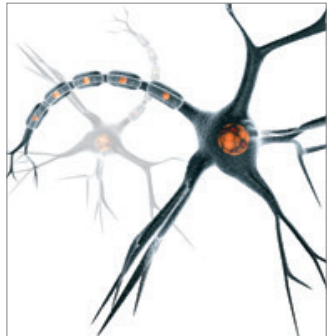

Neural Plasticity
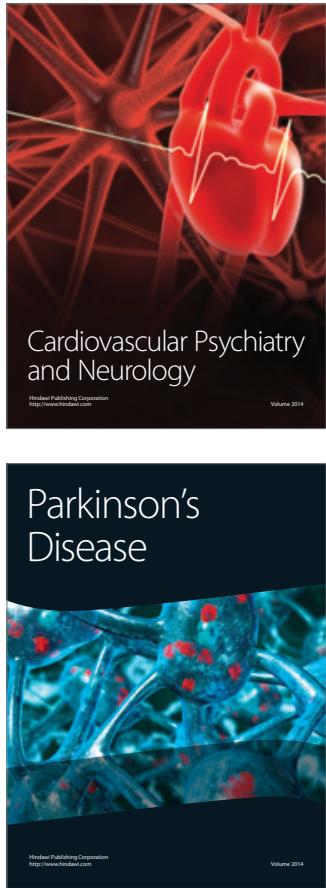